\title{
TEM Studies on Thin Films of Natural Rubber and Polychloroprene Crystallized under Molecular Orientation
}

\author{
Masaki Tsusı, ${ }^{\dagger}$ Toshiki ShImIzu, and Shinzo KoHJIYA \\ Laboratory of Polymer Condensed State, Division of States and Structures III, \\ Institute for Chemical Research, Kyoto University, \\ Uji, Kyoto-fu 611-0011, Japan
}

(Received February 9, 1999)

\begin{abstract}
Thin films of natural rubber (NR) or polychloroprene (CR) were made by casting a $2.0 \mathrm{wt} \%$ solution in benzene onto the water surface, and some of them were prestretched up to a desired amount of strain (mostly, about $200 \%$ ) at ambient temperature. The specimens thus prepared were then crystallized at $-25^{\circ} \mathrm{C}$ for $\mathrm{NR}$ or at $-5^{\circ} \mathrm{C}$ for $\mathrm{CR}$ in a transmission electron microscope (TEM) column with a cryo-transfer specimen-holder and examined by TEM observation without any electron staining. Morphological observations in bright- and dark-field imaging modes and selected-area electron diffraction analysis revealed directly the following: The " $\alpha$-filaments" in NR reported by Andrews [Proc. $R$. Soc. A, 277, 562 (1964)] and the filaments in CR (corresponding to the $\alpha$-filaments in NR) reported by Andrews and Reeve [J. Mater. Sci., 6, 547 (1971)], both of which are observed in the TEM images to orient perpendicularly to the prestretching direction, are undoubtedly identified as edge-on lamellar crystals.

KEY WORDS Natural Rubber/Polychloroprene / Orientation-Induced Crystallization / Morphology / Transmission Electron Microscopy / Dark-Field Image / Defocus Contrast /
\end{abstract}

Crystalline morphologies of natural rubber (NR) were discussed for the first time by Andrews ${ }^{1-3}$ on the basis of the results observed by transmission electron microscopy of its thin films, which had been crystallized isothermally at $-26^{\circ} \mathrm{C}$ (or $-28^{\circ} \mathrm{C}$ ) and most of which had been fixed/stained with osmic acid $\left(\mathrm{OsO}_{4}\right)$ : spherulitic structure in unstrained NR and sheaf-like structure in NR lightly prestretched before crystallization, in both of which " $\alpha$-filaments", named by Andrews, ${ }^{2}$ were arranged in the radial direction. In addition, row (or shish-kebab) structure was observed in uniaxially prestretched NR, in which the $\alpha$-filaments grown perpendicularly to the prestretching direction were stacked in that direction. ${ }^{2}$ Figures $1(\mathrm{a})$-(e) show the schematic crystalline morphologies in NR thin films proposed by Andrews, ${ }^{4}$ which were prestretched up to respective given amounts of strain, $\varepsilon(\%)$. In a series of his experiments, ${ }^{1-3,5} \mathrm{NR}$ thin films cast onto the water surface from a benzene solution were prestretched by a desired $\varepsilon$ at room temperature, crystallized isothermally and then most of them were fixed/stained with $\mathrm{OsO}_{4}$. Based on transmission electron microscope (TEM) investigations of the specimens thus prepared, the resulting morphologies were classified into five groups as shown in Figure 1: An almost similar classification into five groups was given later by Reed, ${ }^{6}$ in which slightly different correlation was, however, proposed between $\varepsilon$ and morphologies.

The morphology observed for $\varepsilon=0 \%$ (no strain) is characterized by the spherulitic structure in which the $\alpha$-filaments are arranged radially (see Figure $1(\mathrm{a})$ ). The morphology for $\varepsilon \fallingdotseq 50 \%$ is well expressed by the sheaflike structure (Figure 1(b)). Figure 1(c) shows the morphology for $\varepsilon \fallingdotseq 100 \%$ corresponding to the structure in which $\alpha$-filaments grown perpendicularly to the prestretching direction are stacked in that direction due to, for example, row nucleation. ${ }^{4,7}$ As shown in Figures 1(c) and $1(d)$, the morphology for $\varepsilon \fallingdotseq 200 \%$ is similar to that for $\varepsilon \fallingdotseq 100 \%$, but the length of $\alpha$-filaments is reduced with an increase in $\varepsilon$. However, the number of rows, each of which consists of the stacked $\alpha$-filaments, increases with increasing $\varepsilon$. The morphologies for $\varepsilon=100-200 \%$ are the same as that expressed as the row or shish-kebab structure. Furthermore, the morphology for $\varepsilon>300 \%$ is expressed by a different structure in which $\alpha$-filaments are not recognized but another type of filaments (termed " $\gamma$-filaments" by Andrews ${ }^{2,3}$ ) can be observed to orient parallel to the prestretching direction (see Figure 1(e)): the $\gamma$-filaments and row nuclei were speculated to be the same species. ${ }^{8}$ Similar morphological results were also reported for crystallized thin films of polychloroprene (CR). ${ }^{9}$ However, there still remain some morphological subjects to be solved, such as to establish the identity of the $\alpha$ - and $\gamma$-filaments in NR and that of the corresponding filamentous entities in CR and to elucidate the mechanism in oriented crystallization at a given amount of strain $(\varepsilon)$.

In this article, morphological studies on the NR and CR thin films which are moderately prestretched outside and then crystallized inside a TEM column, in particular, establishing the identity of the " $\alpha$-filaments" in NR and that of the corresponding filaments in CR will be presented as preliminary results for our future work by in situ TEM observation of the oriented-crystallization process of rubbers.

\section{EXPERIMENTAL}

\section{Materials and Sample Preparation}

Materials used here are NR (Ribbed smoked sheet: RSS\# 1 ) and CR (Neoprene-W, which is a "normal" $\mathrm{CR}^{10}$ containing trans-1,4 (84\%) and cis-1,4 (ca. 10\%) structural units $\left.{ }^{9}\right)$. The procedure for preparing thin films of NR and CR was essentially the same as that reported

+ To whom all correspondence should be addressed (Tel: 0774-38-3061, Fax: 0774-38-3069, e-mail: tsujimas(a scl.kyoto-u.ac.jp). 


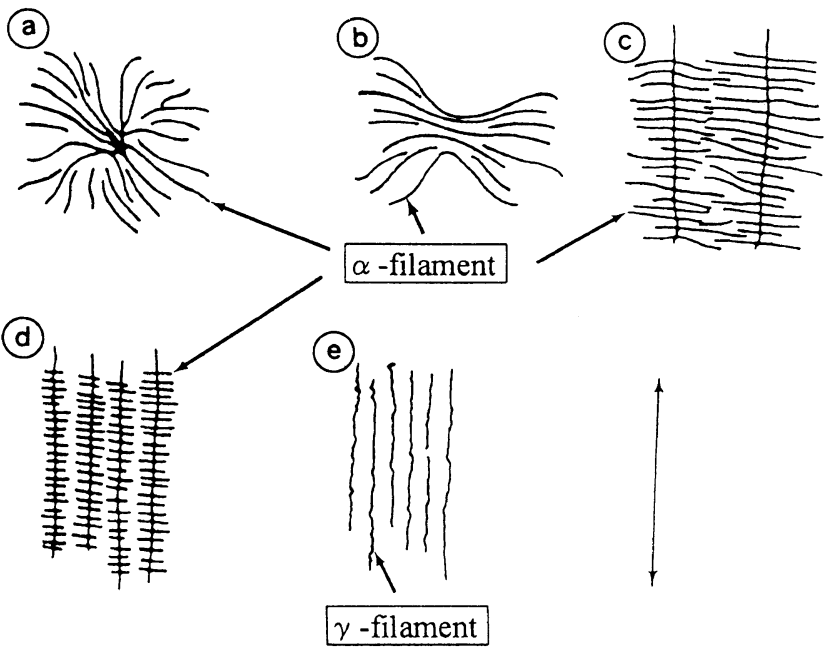

Figure 1. Schematic representation of the crystalline morphologies in NR which were classified into five groups ((a)-(e)) according to the degree of prestretching before crystallization. ${ }^{4}$ The double-headed arrow indicates the prestretching direction (vertical). The amounts of strain, $\varepsilon$ (\%), are as follows: (a) $\varepsilon=0 \%$ (no strain); (b) $\varepsilon \fallingdotseq 50 \%$; (c) $\varepsilon \fallingdotseq 100 \%$; (d) $\varepsilon \fallingdotseq 200 \%$; (e) $\varepsilon>300 \%$.

by Andrews ${ }^{1-3}$ for NR and that by Andrews and Reeve ${ }^{9}$ for CR: Thin films of NR and CR were made by casting each solution $(2.0 \mathrm{wt} \%)$ in benzene onto the water surface. The film thickness was judged from the interference color, and gold-colored portions were selected for subsequent steps. The appropriate portion of the thin film was stretched up to a desired amount of strain $(\varepsilon)$ on the water surface: In order to establish the identity of the " $\alpha$-filaments" in NR and that of the corresponding filaments in $\mathrm{CR}$, the portion was prestretched by a strain of $c a .200 \%$ before crystallization because this amount of strain is to give specimens in which the degree of chain orientation and the length of the filaments in question are both appropriate for our purpose, judging from Figure 1. The prestretched portion of the film was mounted on a carbon-coated copper grid for TEM observation, and then dried. The specimen thus prepared, without any electron staining, was introduced into a TEM column. All these procedures were carried out at ambient temperature in wintertime.

\section{Transmission Electron Microscopy}

A TEM (JEM-200CS, JEOL Ltd.) equipped with a TV system (Model 622SC and 663, Gatan Ltd.) was operated at an accelerating voltage of $160 \mathrm{kV}$ or 200 $\mathrm{kV}$, and this TV system was used to search a suitably crystallized region in the specimen film. A cryo-transfer specimen-holder (EM-CTH11, JEOL Ltd.) was used to cool and crystallize the specimen in the TEM column. The specimen temperature was controlled to be $-25^{\circ} \mathrm{C}$ for NR, at which the growing rate of the $\alpha$-filaments in an NR thin film was reported to be approximately the maximum even in the process of its oriented crystallization, ${ }^{5}$ or to be $-5^{\circ} \mathrm{C}$ for $\mathrm{CR}$, at which the crystallization rate of $\mathrm{CR}$ was reported to be approximately the maximum. ${ }^{11}$ Crystallization time in the TEM column was about $2 \mathrm{~h}$ for the NR film $(\varepsilon \fallingdotseq 200 \%)$, about $7 \mathrm{~h}$ for the unstretched CR film $(\varepsilon=0 \%)$ and less than $1 \mathrm{~h}$ for prestretched CR films $(\varepsilon \fallingdotseq 200$ and $600 \%)$. Bright- and dark-field images, and selected-area electron diffraction (a)

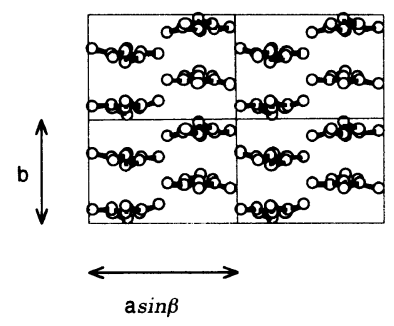

(b)

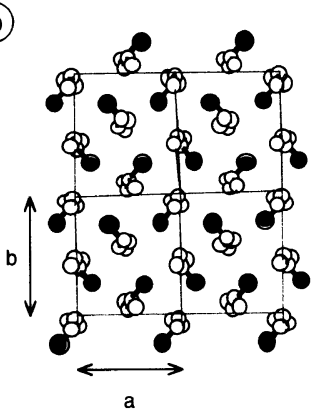

Figure 2. Crystal lattices of NR (a) and of CR (b), viewed along the chain axis $(c$-axis $) .^{13}$ (a) NR: monoclinic $\left(P 2_{1} / a ; a=1.246 \mathrm{~nm}\right.$, $\left.b=0.889 \mathrm{~nm}, c=0.810 \mathrm{~nm}, \beta=92^{\circ}\right)$. (b) CR: orthorhombic $\left(P 22_{1} 2_{1} 2_{1}\right.$; $a=0.884 \mathrm{~nm}, b=1.024 \mathrm{~nm}, c=0.479 \mathrm{~nm})$. In each of the figures, the $c$-axis (chain axis) is normal to the sheet of the figure and pointing upward. Open and closed circles indicate carbon and chlorine atoms, respectively.

(ED) patterns from a specimen area of $c a .8 \mu \mathrm{m}$ in diameter were recorded onto photographic films (Kodak SO-163 or Mitsubishi MEM) which were then developed at $20^{\circ} \mathrm{C}$ for $5 \mathrm{~min}$ with Mitsubishi Gekkol (full strength).

\section{RESULTS AND DISCUSSION}

\section{Natural Rubber (NR)}

A brief survey on the morphological and structural studies on NR was made in our paper on the morphology of NR thin films crystallized under molecular orientation. ${ }^{12}$ The crystal structure of NR, namely of cis-1,4-polyisoprene, was determined by Bunn, ${ }^{13}$ and its crystal system is monoclinic (space group $=P 2_{1} / a$ ): $a=1.246 \mathrm{~nm}, b=0.889 \mathrm{~nm}, c$ (chain axis) $=0.810 \mathrm{~nm}, \beta=$ $92^{\circ}$. Though a different structure for NR crystal ${ }^{14,15}$ was proposed after Bunn, all the ED patterns in the present study are indexed by using the above-mentioned unit cell dimensions. Figure 2(a) shows the projection of the crystal lattice of $\mathrm{NR},{ }^{13}$ viewed along the chain axis (c-axis). Four molecular chains pass through the unit cell. The chains take an efficiently planar conformation in the unit cell, and their assumed molecular planes are all parallel to the crystallographic $a c$-plane. This chain conformation is similar to those in other crystal structures, which were analyzed by Nyburg ${ }^{14}$ and subsequetly by Natta and Corradini ${ }^{15}$ and in which the approximately planar chains are packed in a statistical manner with their molecular planes being all parallel to the crystallographic ac-plane of the orthogonal unit cell.

Figure 3(a) shows an example of frequently observed ED patterns from an NR thin film which was prestretched up to a strain of about $200 \%(\varepsilon \fallingdotseq 200 \%)$ and then crystallized at $-25^{\circ} \mathrm{C}$. The chain axis ( $c$-axis) is found to be oriented rather highly in the prestretching direction (vertical direction of this figure), because the 002 and 004 reflections appear in this direction, namely on the meridian and furthermore the 200 and 400 reflections are concentrated sharply on the equator. In Figure 3(a), the $c$-axis is indicated in the prestretching direction. The $a^{*}$ - and $b^{*}$-axes are, of course, perpendicular to this $c$-axis, but the $c^{*}$-axis is nearly but not exactly parallel to the $c$-axis because of the monoclinic crystal system 


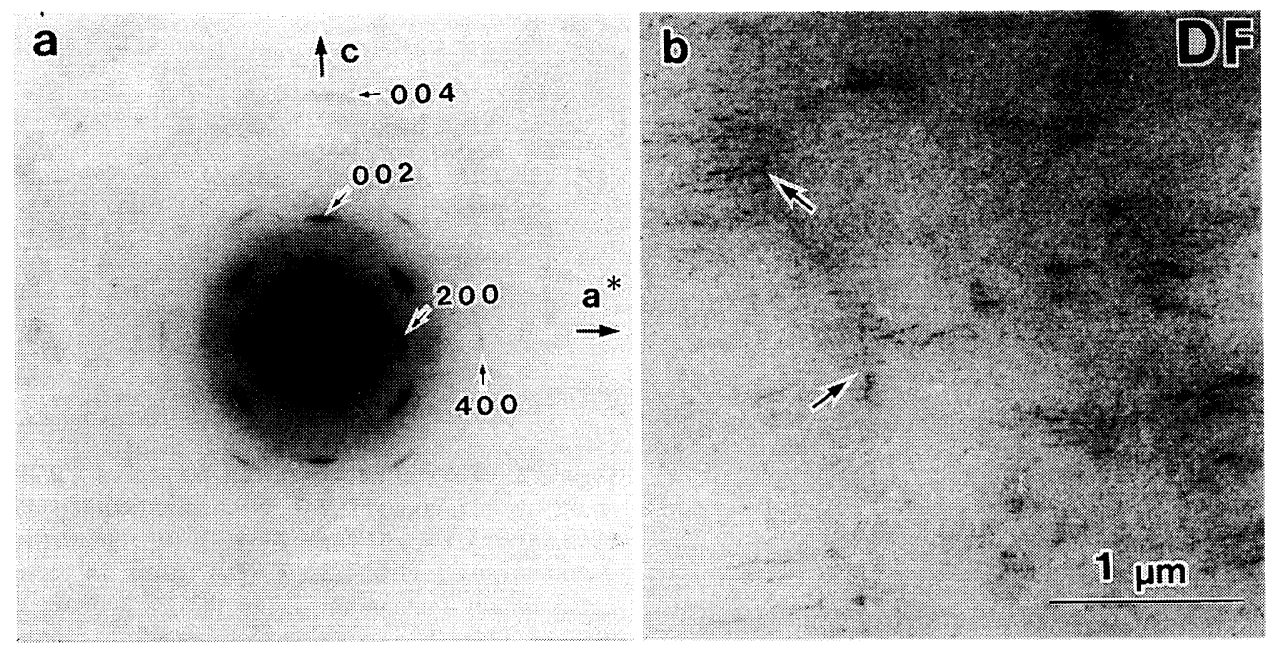

Figure 3. NR thin film which was prestretched up to $\varepsilon \fallingdotseq 200 \%$ and then crystallized at $-25^{\circ} \mathrm{C}$. The prestretching direction is vertical. (a) ED pattern (reversed contrast). The $c$-axis is preferentially oriented in the prestretching direction (vertical). (b) 200 dark-field (DF) image (reversed contrast).

$\left(\beta=92^{\circ}\right)$. The 120 reflection, however, is almost absent, though the absolute value of its structure factor, $\left|F_{120}\right|$, must be fairly greater than that of 200 ; i.e., $\left|F_{120}\right|$ $>\left|F_{200}\right| .^{13,14}$ Absence of the 120 reflection well evidences a so-called double orientation (the uniplanar axial orientation), which was already reported long ago by X-ray diffraction ${ }^{16-18}$ and also recognized by Bunn ${ }^{13}$ and Nyburg: ${ }^{14}$ Nyburg took account of this double orientation in estimating experimentally the structure factors. The molecular planarity of the NR chains in the unit cell might be a major cause for this double orientation in the NR film uniaxially prestretched and subsequently crystallized, as in the case of uniaxial stretching of amorphous films of poly(ethylene 2,6naphthalate) in which the molecular plane defined by the naphthalene rings in the main chain is preferentially oriented parallel to the film surface by uniaxial stretching. ${ }^{19,20}$ It is concluded, therefore, in the NR thin film prestretched up to $\varepsilon \fallingdotseq 200 \%$ that the molecular plane, namely the $(010)$ lattice plane is oriented parallel to the film surface and accordingly the electron beams are incident onto the specimen film in the direction of the $b$-axis.

Figure 3(b) shows the dark-field image (in reversed contrast) taken by using the 200 reflection from an NR thin film (a specimen similar to one used in Figure 3(a)), which was prestretched up to $\varepsilon \fallingdotseq 200 \%$ and then crystallized at $-25^{\circ} \mathrm{C}$. The prestretching direction is vertical. Each dark narrow striation (about $18 \mathrm{~nm}$ in width) in Figure 3(b), which is oriented almost perpendicularly to the prestretching direction, corresponds to a crystalline entity: the entity is to have given at least the 200 reflection in the ED pattern such as Figure 3(a). It is also recognized in Figure 3(b) that such dark filamentous striations are stacked in the prestretching direction. Some typical regions are indicated with arrows. Consequently, it was confirmed that each of these filamentous striations is an edge-on lamella, strictly speaking, its crystalline core, in which the $c$-axis is oriented parallel to the prestretching direction and which was grown in the direction perpendicular to the $c$-axis (viz., in the direction of $a^{*}$-axis), plausibly in the [100] direction (viz., in the direction of $a$-axis). The [100] growing direction of the filamentous entities is the origin of naming them " $\alpha$-filaments", 2 and each of the striations under consideration, of course, must correspond to one $\alpha$-filament. Consequently, the $\alpha$-filaments are undoubtedly identified as the edge-on lamellar crystals. However, the morphology recognized in Figure 3(b) seems to correspond to that for $\varepsilon$ between 100 and $200 \%$ as illustrated in Figures 1(c) and 1(d), though the specimen film was prestretched up to $\varepsilon \fallingdotseq 200 \%$ in our experiment. This discrepancy is probably attributable to partial relaxation of the prestretched film before crystallization.

As was already recognized, the lamellar thickness increases with increasing crystallization temperature $\left(T_{\mathrm{c}}\right)$ in most of the synthetic crystallizable polymers with flexible linear chains such as polyethylene. Therefore, since Andrews ${ }^{4}$ and Reed ${ }^{6}$ proposed the morphological models shown in Figure 1, the $\alpha$-filaments have been assumed to correspond to edge-on crystalline lamellae consisting of folded chains, by combining the results from ED experiments with the fact that the width of the filaments in synthetic cis-1,4-polyisoprene increases with increasing $T_{\mathrm{c}}{ }^{5}$ This assumption, therefore, has been substantiated by our result, as mentioned above.

\section{Polychloroprene (CR)}

The morphological and structural studies on $C R$ were reviewed in our previous paper on crystalline morphologies of CR thin films. ${ }^{21}$ The crystal structure of CR, namely of trans-1,4-polychloroprene, was determined by Bunn, ${ }^{13}$ and its crystal system is orthorhombic (space group $\left.=P 2{ }_{1} 2{ }_{1} 2_{1}\right): a=0.884 \mathrm{~nm}, b=1.024 \mathrm{~nm}, c$ (chain axis) $=0.479 \mathrm{~nm}$. In the present study, all the ED patterns were well indexed by using these unit cell dimensions. Figure 2(b) shows that four molecular chains pass through the unit cell, and also shows no planar chain conformation nor special chain packing which may cause, for example, a double orientation of crystallites: The non-planar chains are located approximately on a twodimensional hexagonal lattice in this $a b$-plane projection. As for crystalline morphologies of CR thin films, 


\section{a}

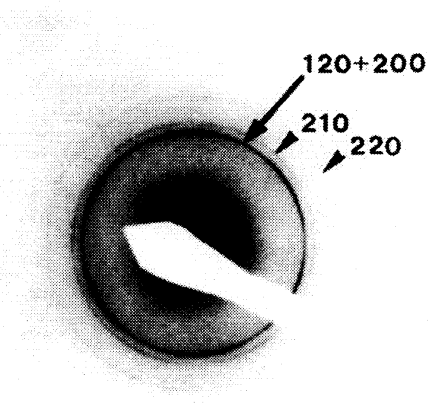

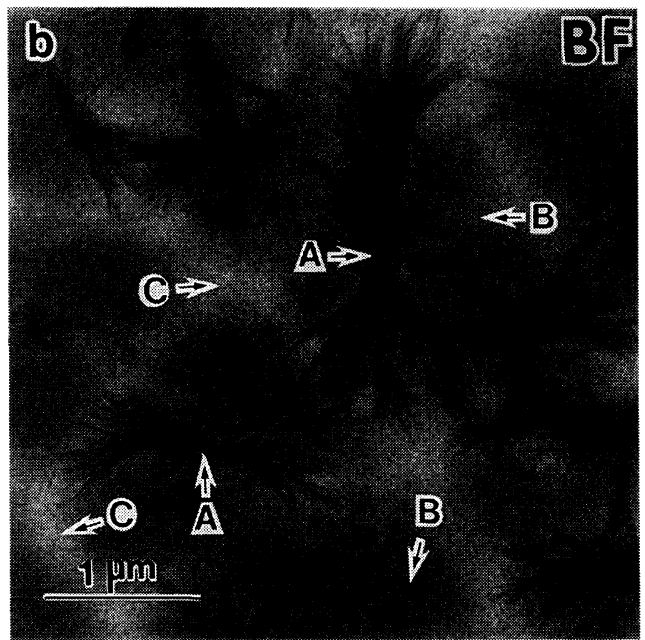

Figure 4. $\mathrm{CR}$ thin film crystallized at $-5^{\circ} \mathrm{C}$ under no strain $(\varepsilon=0 \%)$. (a) ED pattern (reversed contrast). The oblique bright rod is a beam stop. (b) Bright-field (BF) defocus-contrast image of the specimen area used to take (a).

however, only one work by Andrews and Reeve ${ }^{9}$ has been reported so far. In the present study, therefore, the CR thin film crystallized under no strain $(\varepsilon=0 \%)$ was also investigated in order to confirm the morphological result for $\varepsilon=0 \%$ reported by Andrews and Reeve. TEM observations of CR thin films for $\varepsilon=0 \%$ (no strain) and $\varepsilon \fallingdotseq 200 \%$ could be successfully carried out without any electron staining, as in the case of NR mentioned above.

Figure 4(a) is a typical example of the ED patterns obtained from the CR thin film, which was crystallized at $-5^{\circ} \mathrm{C}$ under no strain $(\varepsilon=0 \%)$. This pattern is fairly similar to the wide-angle X-ray diffraction pattern for unoriented $\mathrm{CR}$ which was reported in ref 9. In Figure $4(\mathrm{a})$, the most intense reflection ring is determined as 120 and/or 200, and other two weak rings of 210 and 220 are also observed outside the innermost $120(+200)$ ring. Each of these reflection rings has almost uniform intensity distribution along its Debye-Scherrer circle. Only from this ED pattern, therefore, it would be assumed that most of the crystallites were oriented randomly in the film.

Figure $4(\mathrm{~b})$ is the bright-field image, taken by the defocus contrast method, ${ }^{22}$ of the same area in the CR thin film that was used to take the ED pattern (Figure 4(a)). This figure shows that the texture in our specimen film is essentially similar to that in the films crystallized under no strain in ref 9 . The texture in this figure is composed of three different kinds of domains, namely A-, B-, and C-domains which are indicated, respectively, with the arrows and letters $\mathrm{A}, \mathrm{B}$, and $\mathrm{C}$. The A-domains correspond to sheaf-like structures, namely immature spherulites in which the "filaments" are oriented in the radial direction (in subsequent paragraphs, these "filaments" will be identified as edge-on lamellae). A fully developed spherulitic texture is, however, not recognized in Figure 4(b) though binocularly developed twodimensional spherulites were clearly observed in the film fully crystallized under no strain in ref 9 . The greater population density of crystallization nuclei (possibly, heterogeneous nuclei) and thereby less space for the sheaves (namely, immature spherulites) to mature in our case is deduced to be a probable reason, ${ }^{21}$ because the size of the sheaves in Figure 4(b) is smaller than that in
Figure 1 of ref 9 . Shorter crystallization time $(7 \mathrm{~h})$ in our experiment than that $(7-20 \mathrm{~h})$ in ref 9 might be another reason. The identity of the B-domains is not established in the present study. They, however, seem to correspond to "flat-on" lamellae or at least to nearly flat-on ones. If the ED pattern can be selectively obtained from each of these B-domains in the future work, the structure of the B-domain will be elucidated. The C-domains are apparently the boundaries between adjacent immature spherulites, and correspond to fairly thinner regions than A- and B-domains because the C-domains have a much brighter contrast than the others.

Figure 5(a) is a representative ED pattern from an oriented region in the $\mathrm{CR}$ thin film, which was prestretched up to $\varepsilon \fallingdotseq 200 \%$ and then crystallized at $-5^{\circ} \mathrm{C}$, showing three arc-shaped reflections on the equator. The most intense arc-shaped reflection is determined as 120 and/or 200, and the other weak arcs of 210 and 220 are observed outside the $120(+200)$ arc. Accordingly, the $c$-axis (and also the $c^{*}$-axis) is assumed to be oriented preferentially in the prestretching direction (vertical direction of this figure) though the 002 reflection is not observed on the meridian in Figure 5(a).

Figure 5(b) shows a representative ED pattern from a well-oriented region in the CR thin film prestretched up to $\varepsilon \fallingdotseq 600 \%$ and then crystallized at $-5^{\circ} \mathrm{C}$. In this figure, one arc-shaped reflection, which is determined as 120 and/or 200, is clearly observed on the equator. Faint 210 and 220 reflections, however, are also recognized on the equator. The $c$-axis is found to be highly oriented in the prestretching direction, because the 002 reflection appears on the meridian and in addition, the $120(+200)$ reflection is concentrated more sharply on the equator than that for $\varepsilon \fallingdotseq 200 \%$. Taking this result from Figure 5 (b) into consideration, the $c$-axis should be oriented preferentially in the prestretching direction even in the case of $\varepsilon \fallingdotseq 200 \%$ (see Figure $5(\mathrm{a})$ ) . The reason why no $00 l$ reflections can be observed in the case of $\varepsilon \fallingdotseq 200 \%$ (Figure 5(a)) is deduced to be that the absolute values of the structure factor, $\left|F_{00 l}\right|$, is small, ${ }^{13}$ and also that the degree of chain orientation is low. The degree of chain orientation in the CR specimen, which was prestretched up to $\varepsilon \fallingdotseq 200 \%$ before crystallization, is 

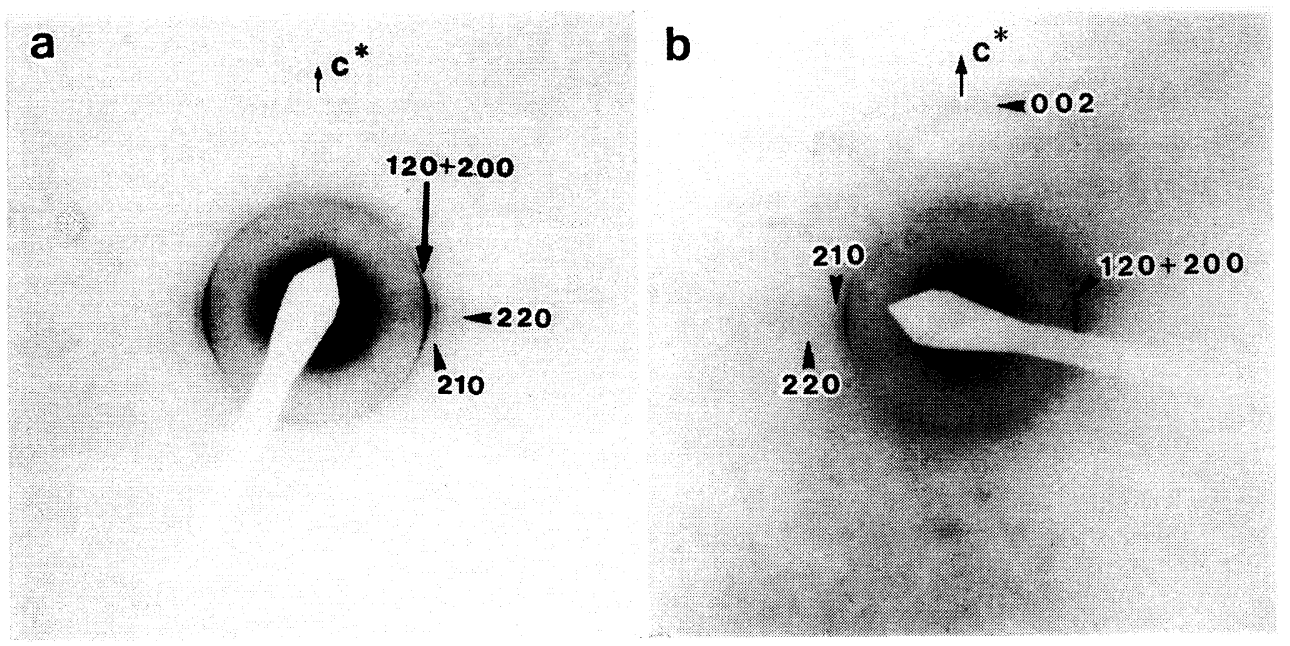

Figure 5. ED patterns from an oriented region in the $\mathrm{CR}$ thin film crystallized at $-5^{\circ} \mathrm{C}$ (reversed contrast). The prestretching direction is vertical, and the oblique bright rod is a beam stop. (a) Prestretched up to $\varepsilon \fallingdotseq 200 \%$. (b) Prestretched up to $\varepsilon \fallingdotseq 600 \%$.
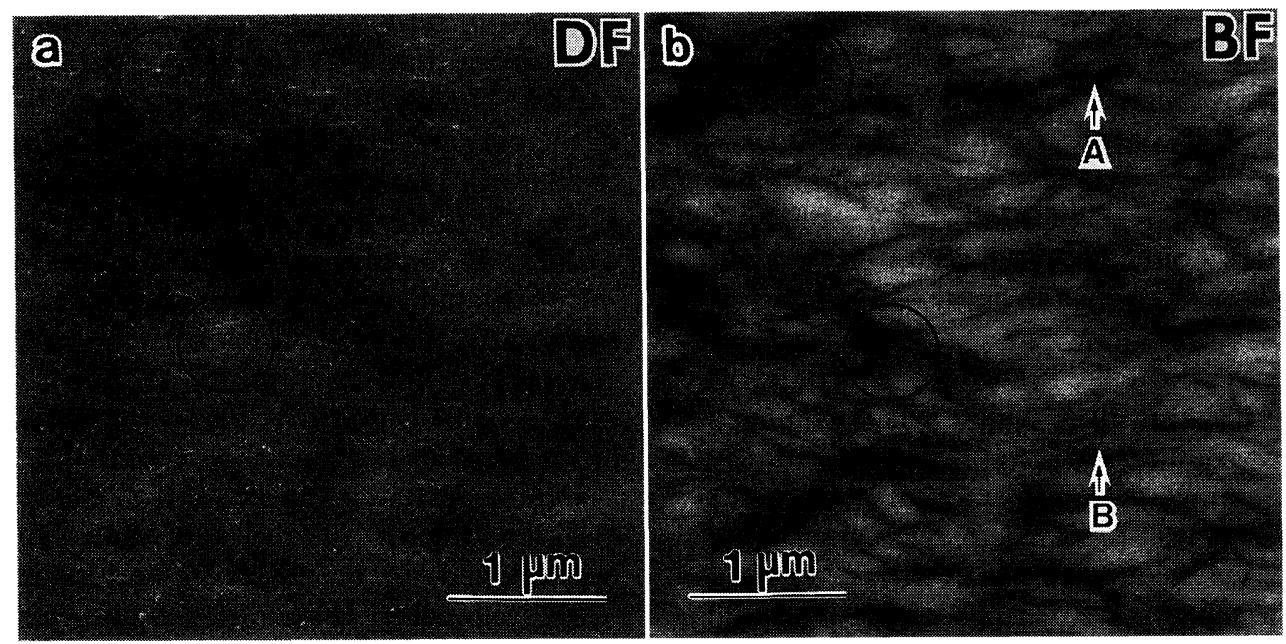

Figure 6. CR thin film which was prestretched up to $\varepsilon \fallingdotseq 200 \%$ and then crystallized at $-5^{\circ} \mathrm{C}$. The prestretching direction is vertical. (a) $120(+200)$ dark-field (DF) image of the specimen area used to take the ED pattern of Figure 5(a). (b) Bright-field (BF) defocus-contrast image of the same specimen area as (a).

lower than that in the above-mentioned NR films prestretched up to $\varepsilon \fallingdotseq 200 \%$ before crystallization (compare Figure 5(a) with Figure 3(a)). Since the average degree of polymerization of CR used here is lower than that of the NR, ${ }^{12,21}$ it is deduced that the number of chain entanglements in CR is smaller than that in NR. It is, therefore, assumed that the stress relaxation in the prestretched CR might take place more rapidly than that in the prestretched NR.

Figure 6(a) is the dark-field image, taken by using the most intense $120(+200)$ reflection on the equator (see Figure 5(a)), from the same region in the CR film that was used to take the ED pattern shown in Figure 5(a). Each of the narrow bright striations (about $10 \mathrm{~nm}$ in width) in Figure 6(a), which are oriented almost perpendicularly to the prestretching direction (vertical direction of this figure), corresponds to a crystalline entity: the entity is to have given at least the 120 or 200 reflection in the ED pattern. It is also recognized that some of such bright filamentous striations are stacked in the prestretching direction in a domain encircled with a black circle. Each of these filamentous striations can be assigned to the edge-on lamella, strictly speaking, its crystalline core, in which the $c$-axis is preferentially oriented parallel to the prestretching direction (viz., perpendicularly to the striation) and which was grown in the direction perpendicular to the prestretching direction.

Figure $6(\mathrm{~b})$ is the bright-field image, taken by the defocus contrast method, from the same region in the CR film used in taking Figures 5(a) and 6(a). The whole area of Figure $6(\mathrm{~b})$ corresponds to that of Figure 6(a). Figure 6(b) reveals that dark curved striations, namely wavy "filaments" are oriented almost perpendicularly to the prestretching direction. Some of them are arranged to make a sheaf-like structure (indicated with the arrow and a letter A), and some are stacked in the prestretching direction to make a row (or shish-kebab) structure (indicated with the arrow and a letter B). Black circles in Figure 6(b) indicate respectively the same areas that were encircled in Figure 6(a), and each of these areas corresponds to a part of a row (or shish-kebab) structure. Combined with the result of Figure 6(a), these "filaments" observed in Figure 6(b) are, consequently, 
identified as the edge-on lamellae, as concluded above for the " $\alpha$-filaments" in NR. The "filaments" in Figure 6(b) are stacked to make so-called row (or shish-kebab) structure as a whole. The degree of chain orientation in this specimen film $(\varepsilon \fallingdotseq 200 \%)$ is, however, much lower than that obtained for $\varepsilon \fallingdotseq 200 \%$ by Andrews and Reeve ${ }^{9}$ but rather similar to that observed for $\varepsilon \fallingdotseq 100 \%$ by them. Though we utilized here same "Neoprene-W" as they did in their experiment, such a morphological difference occurred. The stress relaxation of the prestretched CR thin film before crystallization and TEM observation, for example during drying of the film, might be one of the principal factors which are responsible for the morphological difference. Some unknown slight difference in composition ratio of the trans-1,4 and cis-1,4 constituents between our "Neoprene-W" and their one might be another factor. The prestretching rate seems to be also another important factor responsible for the morphological difference. ${ }^{21}$

\section{CONCLUSIONS}

1. In the dark-field image taken by using the 200 reflection for the NR film prestretched up to $\varepsilon \fallingdotseq 200 \%$, each of the narrow striations which were oriented almost perpendicularly to the prestretching direction could be identified as an edge-on lamellar crystal.

In the dark-field image taken by using the $120(+200)$ reflection for the CR film prestretched up to $\varepsilon \fallingdotseq 200 \%$, each of the narrow striations which were oriented almost perpendicularly to the prestretching direction could also be identified as an edge-on lamellar crystal. Hence, the wavy "filaments" in the CR film, which were oriented almost perpendicularly to the prestretching direction in the bright-field defocus-contrast image of the same specimen area used in taking the dark-field image, were well ascertained to be edge-on crystalline lamellae.

Consequently, each of the " $\alpha$-filaments" in NR and corresponding filaments in $\mathrm{CR}$ was identified as an edge-on crystalline lamella.

2. In orientation-induced crystallization of flexible linear polymers from the melt, the row structure or so-called shish-kebab structure will be formed. ${ }^{23-25}$ The morphologies for the specimens prestretched up to $\varepsilon \fallingdotseq 200 \%$, as demonstrated in Figures 3 and 6 , might be called the row structure because "shish" crystals were not clearly recognized in these figures.

3. TEM observation of crystalline morphologies in the thin films of NR and CR without any electron staining was successfully carried out in the present study, and thus our future in situ TEM study on rubbers during oriented crystallization in a TEM column has become promissing. However, there still remain some hurdles to overcome, for example, electron irradiation damage to the specimens.

This research was presented in part at the Regional Conference on Polymeric Material '98 (Universiti Sains Malaysia, Penang; November 10-11, 1998).

Acknowledgments. The authors would like to express their grateful acknowledgment to Aburahi Laboratories, Shionogi \& Co., Ltd. for permission to use the cryotransfer specimen-holder (EM-CTH11). This work was supported by a Grant-in-Aid for Scientific Research (No. 10650886) from the Ministry of Education, Science, Sports and Culture of Japan and partly by a grant (in 1996) from Eno Science Foundation, to both of which M.T. wishes to express his gratitude.

\section{REFERENCES}

1. E. H. Andrews, Proc. R. Soc. A, 270, 232 (1962).

2. E. H. Andrews, Proc. R. Soc. A, 277, 562 (1964).

3. E. H. Andrews, J. Polym. Sci., 4, 668 (1966).

4. E. H. Andrews, Pure Appl. Chem., 31, 91 (1972).

5. E. H. Andrews, P. J. Owen, and A. Singh, Proc. R. Soc. London A, 324, 79 (1971); Rubber Chem. Technol., 45, 1315 (1972).

6. P. E. Reed, Proc. R. Soc. London A, 338, 459 (1974).

7. B. C. Edwards, J. Polym. Sci., Polym. Phys. Ed., 13, 1387 (1975).

8. B. C. Edwards and P. J. Phillips, J. Polym. Sci., Polym. Phys. Ed., 13, 2117 (1975).

9. E. H. Andrews and B. Reeve, J. Mater. Sci., 6, 547 (1971).

10. J. T. Maynard and W. E. Mochel, J. Polym. Sci., 18, 227 (1955).

11. V. Grmela and L. Megarskaya, Polym. Sci. USSR, 10, 1196 (1968).

12. T. Shimizu, M. Tsuji, and S. Kohjiya, Mater. Sci. Res. Intl., 4, 117 (1998).

13. C. W. Bunn, Proc. R. Soc. A, 180, 40 (1942).

14. S. C. Nyburg, Acta Crystallogr., 7, 385 (1954).

15. G. Natta and P. Corradini, Angew. Chem., 68, 615 (1956); Nuovo Cimento Suppl., 15, 111 (1960).

16. H. Mark and G. V. Susich, Kolloid-Z., 46, 11 (1928).

17. H. A. Morss, Jr., J. Am. Chem. Soc., 60, 237 (1938).

18. S. D. Gehman and J. E. Field, J. Appl. Phys., 10, 564 (1939).

19. S. Murakami, Y. Nishikawa, M. Tsuji, A. Kawaguchi, S. Kohjiya, and M. Cakmak, Polymer, 36, 291 (1995).

20. S. Murakami, M. Yamakawa, M. Tsuji, and S. Kohjiya, Polymer, 37, 3945 (1996).

21. T. Shimizu, M. Tsuji, and S. Kohjiya, J. Mater. Res., 14, 1645 (1999).

22. J. Petermann and H. Gleiter, Phil. Mag., 31, 929 (1975).

23. M. J. Hill and A. Keller, J. Macromol. Sci.-Phys., B3, 153 (1969).

24. T. Amano, S. Kajita, and K. Katayama, Progr. Colloid Polym. Sci., 58, 108 (1975).

25. K. Katayama, Sen-i Gakkaishi, 34, P-63 (1978). 